\title{
Cross-section measurements for the formation of manganese-52 and its isolation with a non-hazardous eluent
}

\author{
By M. Buchholz, I. Spahn*, B. Scholten and H. H. Coenen \\ Institut für Neurowissenschaften und Medizin, INM-5: Nuklearchemie, Forschungszentrum Jülich GmbH, D-52425 Jülich, Germany
}

(Received January 25, 2013; accepted in revised form April 23, 2013)

(Published online July 29, 2013)

\begin{abstract}
Nuclear reaction / Cross section / Thick target yield / Integral yield / Separation of positron emitter ${ }^{52} \mathrm{Mn} /$ Ion exchange chromatography
\end{abstract}

Summary. With respect to the production of no-carrieradded ${ }^{52} \mathrm{Mn}$ nuclear reactions on natural chromium were investigated. Cross sections of the reactions ${ }^{\text {nat }} \mathrm{Cr}(p, x)^{48} \mathrm{~V}$, ${ }^{48,49,51} \mathrm{Cr}$, ${ }^{52 \mathrm{~g}, \mathrm{~m}} \mathrm{Mn}$ were determined in the proton energy range of 7.6 to $45 \mathrm{MeV}$. Additionally, production yields of ${ }^{52 \mathrm{~g}, \mathrm{~m}} \mathrm{Mn}$ and ${ }^{51} \mathrm{Cr}$ were measured in the energy range from 8.2 to $16.9 \mathrm{MeV}$ and therefrom the calculated saturation thick target yields were obtained as $(2.55 \pm 0.31),(6.96 \pm 0.57)$, and $(1.53 \pm 0.15) \mathrm{GBq} / \mu \mathrm{A}$, respectively.

For in vivo applications like PET, low toxicity is critical and sufficient activity of a radiolabelled compound mandatory. Thus, additional purification steps after separation of radionuclides and target materials have to be avoided. However, no isolation procedure has been reported in the literature so far where radiomanganese is directly obtained in a nonhazardous solution. Therefore a new separation procedure was developed utilizing the cation-exchange resin DOWEX $50 \mathrm{~W} \times 8 \quad\left(\mathrm{H}^{+}\right.$-form $) .{ }^{52 \mathrm{~g}} \mathrm{Mn}$ was quantitatively isolated from "bulk" chromium after 3 to $4 \mathrm{~h}$ in non-hazardous $0.067 \mathrm{M}$ ammonium citrate solution. Up to $99 \%$ of ${ }^{52 \mathrm{~g}} \mathrm{Mn}$ activity was harvested within 10 to $15 \mathrm{~mL}$ eluent solution with no measureable ${ }^{51} \mathrm{Cr}$ impurities.

\section{Introduction}

Production of positron emitting radioisotopes of manganese is important in the development of multimodal tracers for medical imaging. Due to the possible quantitation of low concentrations of labeled tracers with positron emission tomography (PET) and the high morphological resolution of magnetic resonance imaging (MRI) it is of high interest to develop multimodal imaging probes and thereby utilize the full potential of new PET/MRI devices [1]. This is especially promising for the evaluation of new MRI contrast agents. Aspects to be evaluated include among others: the overall body distribution, the distribution time needed and the biological half-life.

Positive contrast agents in MRI are up to now mainly based on two elements: gadolinium and manganese. Gado-

\footnotetext{
*Author for correspondence (E-mail: i.spahn@fz-juelich.de).
}

linium-based contrast agents occasionally lead to the occurrence of nephrogenic systemic fibrosis (NSF) [2,3], but show a better performance than manganese based contrast agents [4]. Those have rather been neglected, due to toxicity of free manganese [5]. Therefore, research was recently directed back to manganese compounds avoiding NSF.

An authentic radiolabelling of these new manganese contrast agents is preferable, because ligand or shell labelled compounds may be metabolised and lead to false distribution and excretion information on the toxic core element. Generally used standard PET radionuclides $\left({ }^{18} \mathrm{~F},{ }^{11} \mathrm{C},{ }^{124} \mathrm{I}\right)$ allow here no authentic labelling. Therefore development of positron emitting radioisotopes of manganese appears important. Two radioisotopes, namely ${ }^{51} \mathrm{Mn}\left(T_{1 / 2}=46.2 \mathrm{~min}\right.$, $\beta^{+}$-decay intensity: $96.86 \%$, max. $\beta^{+}$-energy: $2185 \mathrm{keV}$ ) and ${ }^{52 \mathrm{~g}} \mathrm{Mn}\left(T_{1 / 2}=5.6 \mathrm{~d}, \beta^{+}\right.$-decay intensity: $29.6 \%$, max. $\beta^{+}$-energy: $575 \mathrm{keV}$ ) are promising candidates. Extensive studies have been carried out in our Institute on ${ }^{51} \mathrm{Mn}$ [6-9]. In this work we concentrate on ${ }^{52} \mathrm{Mn}$. Proton induced nuclear reaction cross sections on natural chromium were measured in the energy range from 7.6 up to $45 \mathrm{MeV}$ leading to the formation of the positron emitting isomers ${ }^{52 \mathrm{~g}, \mathrm{~m}} \mathrm{Mn}$ and the by-products ${ }^{48} \mathrm{~V}$ and ${ }^{48,49,51} \mathrm{Cr}$. A measurement of thick target yields was done to optimize the production of ${ }^{52 \mathrm{~g}, \mathrm{~m}} \mathrm{Mn}$ and ${ }^{51} \mathrm{Cr}$ in the proton energy range from 8.2 to 16.9 MeV ( ${ }^{52 \mathrm{~g}} \mathrm{Mn}$ excitation function maximum). According to procedures reported so far a subsequent processing step is mandatory directly after the separation of radiomanganese from the bulk target material, due to the use of either undesirable co-precipitants $\left(\mathrm{Al}(\mathrm{OH})_{3}\right)$ [10], inactive manganese carrier [11] or toxic and/or aggressive solvents (i.e. concentrated $\mathrm{HCl}$ ) [8]. Therefore a new separation method using ion-exchange chromatography was developed leading to nocarrier-added radiomanganese in a suitable non-hazardous medium for later radioactive labelling of contrast agents.

\section{Experimental}

\subsection{Target sample preparation}

The preparation of thin and homogenous chromium targets was mandatory for cross section measurements. Relatively thin foils of elemental chromium are not commercially available, due to its brittle nature. Therefore thin target layers 
were prepared by an electro-deposition method of Fessler et al. [12], as a newer electro-deposition method by Klein et al. [6] did not prove to be reproducible.

Natural chromium was electrolytically deposited on a $0.01 \mathrm{~mm}$ thick gold backing ( $99.9 \%$ purity, Goodfellow) with $13 \mathrm{~mm}$ in diameter. The electro-deposition cell [13] consisted of a copper cathode, covered by the changeable gold foil and a planar platinum foil anode. A 1.4 M chromic acid solution (chromium(VI) oxide, 99.99\% Sigma Aldrich) with a $0.02 \mathrm{M}$ sulfuric acid additive (Merck) served as the electrolyte in the central glass cylinder. The chromium layer was deposited at a current density of $140-165 \mathrm{~mA} / \mathrm{cm}^{2}$ and a voltage of $3.5 \mathrm{~V}$. In all experiments the platinum anode was slowly rotated in order to achieve highest possible homogeneity. For preparation of thicker chromium targets the electrolyte was renewed after $15 \mathrm{~min}$ in order to sustain a constant deposition speed on the gold foil. The target layers produced consisted of $0.004-0.013 \mathrm{~g} / \mathrm{cm}^{2}$ elemental chromium. Their chemical purity was determined by ICPOES analysis of the deposited layer and was found to be $98.6 \pm 3.2 \%$.

In addition to electrolytically deposited chromium targets, sediments of $\mathrm{Cr}_{2} \mathrm{O}_{3}$ (p.a. grade purity, Sigma Aldrich) were prepared on aluminium backings by the batch sedimentation technique [14]. The aluminium backings $(99.9 \%$ purity, Goodfellow) were $15 \mathrm{~mm}$ in diameter and $50 \mu \mathrm{m}$ thick. The sediments were prepared by slow evaporation of the solvent from an ethanolic suspension at room temperature and subsequent drying at $50{ }^{\circ} \mathrm{C}$ for 3 to $8 \mathrm{~h}$. They were then covered with aluminium foils $(99.9 \%$ purity, Goodfellow) of $10 \mu \mathrm{m}$ thickness. The weight of the sediments (13 $\mathrm{mm}$ diameter) ranged between 0.008 and $0.030 \mathrm{~g} / \mathrm{cm}^{2}$. The used Teflon sedimentation chamber has been described in detail [15] and was slightly adapted in its dimensions in this work.

For thick target yield measurements and upscale studies of the present separation round targets (thickness: $1 \mathrm{~mm}$, diameter: $13 \mathrm{~mm}$ ) were wire eroded from cast disks of natural chromium (Goodfellow Ltd.) with a purity of $99.9 \%$.

\subsection{Irradiations, beam and proton energy analysis}

\subsubsection{Irradiations}

The cross section determination was conducted using the stacked-foil technique. Each stack consisted of four to seven chromium targets, additional monitor foils, aluminum absorbers and catcher foils. All monitors and absorbers were high-purity metal foils provided by Goodfellow Ltd. The thin Al catcher foils $(10 \mu \mathrm{m})$ were implemented between targets and monitor foils to catch eventually ejected monitor nuclides. The foil stacks were irradiated at the Baby Cyclotron BC1710 or at the cyclotron JULIC, both at the Forschungszentrum Jülich, in the proton energy range of 17 to $6 \mathrm{MeV}$ and 45 to $15 \mathrm{MeV}$, respectively. At the Baby Cyclotron a beam current of 0.5 to $1.0 \mu \mathrm{A}$ was used and the irradiation time was 0.5 to $1 \mathrm{~h}$, while irradiations at the cyclotron JULIC were conducted with a beam current of 0.2 to $0.5 \mu \mathrm{A}$ for 2 to $3 \mathrm{~h}$.

A stack of cast chromium plates with copper monitor foils (10 $\mu \mathrm{m}$ thickness) and a copper beam stop for adequate thermal conductivity was irradiated at the Baby Cyclotron $\mathrm{BC} 1710$ to measure experimental production rates and determine saturated thick target yields. The irradiation was done with a beam current of $1 \mu \mathrm{A}$ for $1 \mathrm{~min}$.

\subsubsection{Beam analysis}

The beam currents were measured with a beam integrator Faraday Cup $( \pm 1 \mu \mathrm{C}$ uncertainty). For precise determination of the average beam current throughout the stack, monitor reactions were used. In the energy range between 45 and $20 \mathrm{MeV}$ nickel foils were used, whereas in the lower energy range between 20 and $6 \mathrm{MeV}$ titanium and copper foils were implemented in the foil stacks. The respective monitor reactions are ${ }^{\text {nat }} \mathrm{Ni}(p, x)^{57} \mathrm{Ni}$, ${ }^{\text {nat }} \mathrm{Ti}(p, x)^{48} \mathrm{~V}$ and ${ }^{n a t} \mathrm{Cu}(p, x)^{62,63,65} \mathrm{Zn}$. The monitor radionuclides produced, their half-lives and the $\gamma$-rays used are given in Table 1 . The proton flux density and thus the beam current were calculated from the induced radioactivity of the monitor nuclides using the activation formula and the recommended cross sections for the nuclear reactions taken from the Nuclear Data Evaluated File of the IAEA [16].

After a sufficient decay time the monitor foils in the back and front of the foil stacks were also analysed with an instant imager (Packard Instant Imager), which enables determination of the spatial distribution of the radioactivity by utilization of small Geiger-Müller counters covering the observed area. This allowed the evaluation of the beam profile including position and size of the proton beam created at both cyclotrons. Both beams were adequately focused for the targets used (JULIC: diameter $\approx 4 \mathrm{~mm}$; BC1710: diameter $\approx 6 \mathrm{~mm}$ ). The beam profile of protons at the JULIC cyclotron was evaluated in more detail earlier [17].

\subsubsection{Proton energy analysis}

The proton energy degradation was calculated beforehand by the Excel-routine STACK of the Forschungszentrum

Table 1. Half-lives and $\gamma$-lines with intensities of measured radionuclides, taken from Ref. [20].

\begin{tabular}{lccc}
\hline Isotope & Half-life & $\gamma$-line $[\mathrm{keV}]$ & Intensity [\%] \\
\hline${ }^{48} \mathrm{~V}$ & $(15.974 \pm 0.003) \mathrm{d}$ & $983.517 \pm 0.005$ & $99.99 \pm 0.2$ \\
& & $1312.096 \pm 0.006$ & $975 \pm 0.8$ \\
${ }^{48} \mathrm{Cr}$ & $(21.56 \pm 0.03) \mathrm{h}$ & $112.36 \pm 0.06$ & $96.0 \pm 0.6$ \\
& & $308.25 \pm 0.05$ & 100 \\
${ }^{49} \mathrm{Cr}$ & $(42.3 \pm 0.1) \mathrm{min}$ & $62.289 \pm 0.002$ & $16.39 \pm 0.21$ \\
& & $90.639 \pm 0.002$ & $53.20 \pm 0.11$ \\
& & $152.928 \pm 0.002$ & $30.32 \pm 0.11$ \\
${ }^{51} \mathrm{Cr}$ & $(27.703 \pm 0.002) \mathrm{d}$ & $320.0824 \pm 0.0004$ & $9.91 \pm 0.01$ \\
${ }^{52 \mathrm{~g}} \mathrm{Mn}$ & $(5.591 \pm 0.003) \mathrm{d}$ & $744.233 \pm 0.013$ & $90.0 \pm 0.8$ \\
& & $935.538 \pm 0.011$ & $94.5 \pm 0.9$ \\
& & $1434.068 \pm 0.014$ & $100.0 \pm 0.5$ \\
${ }^{52 \mathrm{~m}} \mathrm{Mn}$ & $(21.1 \pm 0.2) \mathrm{min}$ & $377.748 \pm 0.005$ & 1.7 \\
& & $1434.068 \pm 0.014$ & $98.3 \pm 2.0$ \\
${ }^{57} \mathrm{Ni}$ & $(35.60 \pm 0.06) \mathrm{h}$ & $127.164 \pm 0.003$ & $129 \pm 0.3$ \\
& & $1377.63 \pm 0.03$ & $779 \pm 1.6$ \\
${ }^{62} \mathrm{Zn}$ & $(9.186 \pm 0.013) \mathrm{h}$ & $548.38 \pm 0.11$ & $15.2 \pm 1.4$ \\
& & $596.70 \pm 0.13$ & 26.0 \\
${ }^{63} \mathrm{Zn}$ & $(38.47 \pm 0.05) \mathrm{min}$ & $669.62 \pm 0.05$ & $8.4 \pm 0.1$ \\
& & $962.06 \pm 0.04$ & $6.6 \pm 0.4$ \\
${ }^{65} \mathrm{Zn}$ & $(244.26 \pm 0.26) \mathrm{d}$ & $1115.546 \pm 0.004$ & $50.75 \pm 0.24$ \\
\hline
\end{tabular}


Jülich, which is based on the Bethe-Bloch formalism [18] including the adjustments of Williamson et al. [19]. The above mentioned monitor reactions were additionally used to control the energy of the degraded beam.

The monitor foils were compliant within the rising standard uncertainty of the Excel-routine STACK $( \pm 0.1 \mathrm{MeV}$ per degraded $\mathrm{MeV}$ ) over the complete energy range. The incident proton energy of JULIC was evaluated in detail earlier [17] showing an incident energy uncertainty of $0.2 \mathrm{MeV}$. The incident proton energy of BC1710 was evaluated experimentally in this work by three pure monitor stacks using the cross section ratios of several monitor nuclides $\left({ }^{48} \mathrm{~V}\right.$, $\left.{ }^{62,63,65} \mathrm{Zn}\right)$. The experimentally determined incident proton energy was $E=17.2 \pm 0.1 \mathrm{MeV}$.

\subsection{Cross section determination and thick target yield $\gamma$-ray measurements}

All $\gamma$-ray measurements of activated targets and monitor foils were performed using high purity germanium detectors (HPGe) which were energy and efficiency calibrated using certified, standard radiation point sources of ${ }^{226} \mathrm{Ra},{ }^{152} \mathrm{Eu}$, ${ }^{133} \mathrm{Ba},{ }^{241} \mathrm{Am}$ and ${ }^{60} \mathrm{Co}$ (Amersham Ltd. and PhysikalischTechnische Bundesanstalt Braunschweig). In the proton energy range of 7.6 to $30 \mathrm{MeV}$ the irradiated chromium targets were measured non-destructively. In the higher energy region the activation of the gold-backing was too strong for a direct, non-destructive $\gamma$-ray measurement. Therefore the chromium layers were separated from the gold-foils by dissolving in $2-3 \mathrm{~mL}$ of hot $\left(70^{\circ} \mathrm{C}\right)$, fuming hydrochloric acid and a subsequent measurement by $\gamma$-ray spectroscopy was carried out. The resulting solutions were measured in glass vials $2 \mathrm{~cm}$ in diameter. These measurements were conducted at a distance of $50 \mathrm{~cm}$ from the HPGe-detector heads in order to minimize efficiency deviations due to differing detection geometries from the calibration point sources. The $\mathrm{Cr}_{2} \mathrm{O}_{3}$ sediment targets could be measured non-destructively over the whole proton energy range.

\subsection{1 ${ }^{52 \mathrm{~m}}$ Mn activity measurements and cross section calculations}

The $\gamma$-rays used to calculate the absolute radioactivity of the radionuclides formed are also given in Table 1. The undisturbed $377 \mathrm{keV} \gamma$-line of the short-lived radionuclide ${ }^{52 \mathrm{~m}} \mathrm{Mn}$ $\left(T_{1 / 2}=21.1 \mathrm{~min}\right)$ has a low intensity of $1.7 \%$. The more intense $\gamma$-line at $1434 \mathrm{keV}(98.3 \%)$ is interfered by the identical $\gamma$-line of the longer-lived ${ }^{52 \mathrm{~g}} \mathrm{Mn}$ isotope (100\%). Neither the subtraction of $\gamma$-ray spectra obtained before and after a complete decay of ${ }^{52 \mathrm{~m}} \mathrm{Mn}$, nor the subtraction of the activity obtained from the undisturbed $\gamma$-ray $(743 \mathrm{keV})$ of ${ }^{52 \mathrm{~g}} \mathrm{Mn}$ from the interfering $\gamma$-ray $(1434 \mathrm{keV})$ yielded consistent results for the activity of ${ }^{52 \mathrm{~m}} \mathrm{Mn}$. In order to solve this problem, samples were measured repeatedly over 10 half-lives of the metastable ${ }^{52 \mathrm{~m}} \mathrm{Mn}$ after EOB. The measurement times were subsequently raised from 0.5 to $6 \mathrm{~h}$ and additional $\gamma$-ray spectra were recorded over a period of $2-3 \mathrm{~d}$. The resulting decay curve was then used to extrapolate the radioactivity of the short-lived radioisotope ${ }^{52 \mathrm{~m}} \mathrm{Mn}$ for the end of bombardment. The activity uncertainty depended mainly on the goodness of fit and was estimated to be about $5 \%$. The cross sections were calculated using the activation formula and the experimentally measured radioactivity.

\subsection{2 ${ }^{52 \mathrm{~g}} \mathrm{Mn},{ }^{48} \mathrm{~V}$, and ${ }^{48,49,51} \mathrm{Cr}$ activity measurements}

Due to the isomeric transition of ${ }^{52 \mathrm{~m}} \mathrm{Mn}(1.75 \pm 0.05 \%)$ to

${ }^{52 \mathrm{~g}} \mathrm{Mn}$ only cumulative activities of ${ }^{52 \mathrm{~g}} \mathrm{Mn}$ could be measured directly. The $\gamma$-ray measurements for the activity assessment of ${ }^{52 \mathrm{~g}} \mathrm{Mn}$ were therefore conducted at least one day after EOB when all ${ }^{52 \mathrm{~m}} \mathrm{Mn}$ had decayed out. The major $\gamma$-lines given in Table 1 were employed to calculate the absolute activity which coincided in general in the limit of $1 \sigma$. The average value of the absolute activity was used to obtain the respective cumulative cross section of ${ }^{52 \mathrm{~g}} \mathrm{Mn}$.

The activities of the by-products ${ }^{48,49} \mathrm{Cr}$ were deduced from spectra obtained in short measurements of the ${ }^{52 \mathrm{~m}} \mathrm{Mn}$ activity and those of ${ }^{48} \mathrm{~V}$ and ${ }^{51} \mathrm{Cr}$ in the extended determination of the cumulative ${ }^{52 \mathrm{~g}} \mathrm{Mn}$ activity, corresponding to the half-life of the respective radioisotope. The absolute activities were calculated from the most intense $\gamma$-lines (Table 1).

\subsubsection{Experimental and calculated integral yields}

The thick target yield over the proton energy range of $16.9 \mathrm{MeV}$ to $8.2 \mathrm{MeV}$ was experimentally determined to cover full excitation function of the nuclear reactions ${ }^{\text {nat }} \mathrm{Cr}(p, x)^{52 \mathrm{~g}, \mathrm{~m}} \mathrm{Mn}$. As mentioned before, the target after the short irradiation was repeatedly measured non-destructively, beginning $63 \mathrm{~min}$ (three half-lives of ${ }^{52 \mathrm{~m}} \mathrm{Mn}$ ) after EOB. With the obtained radioactivities the production rates were calculated. The saturation thick target yield could be then arithmetically obtained by extrapolation from the production rates by the formula Eq. (1):

$$
\mathrm{TTY}_{\text {saturation }}=\frac{A \cdot\left(1-\mathrm{e}^{-\lambda \cdot 10 \cdot T_{1 / 2}}\right)}{I \cdot\left(1-\mathrm{e}^{-\lambda \cdot t_{\text {irr }}}\right)}
$$

with $A$ : measured activity, $\lambda$ : decay constant of the corresponding nuclide, $T_{1 / 2}$ : half-life of the corresponding nuclide in sec, $I$ : proton current in $\mu \mathrm{A}$, and $t_{\text {irr }}$ : irradiation time in sec.

The theoretical integral thick target yields were calculated from the measured excitation functions using the Stack routine and the activation formula over the corresponding energy range. The results were compared with the experimental values.

\subsubsection{Uncertainties}

The main uncertainties in the measured cross sections and thick target yields described above are as follows:

- individual uncertainties of area weight determination of foils (1-3\%) derived from weighing and area uncertainties.

- particle flux determination (5-10\%) due to experimental measurement uncertainties and uncertainties in literature cross sections of monitor reactions [16].

- individual uncertainties of the detector efficiency (3$20 \%$ ) due to the limited coverage of standard radiation sources over the whole $\gamma$-ray energy region measured.

- statistical uncertainty of peak area $(0.5-10 \%)$ due to Gaussian-shaped peak fitting with strongly varying 
counting statistics, dependent on activity, $\gamma$-ray intensity and efficiency of the detector for the $\gamma$-ray energy.

$-\gamma$-ray intensity uncertainty $(0.5-1.5 \%)$ taken from literature [20].

With additional minor errors (e.g. in molar weight, half-life etc.) those lead to the individual uncertainties calculated according to Gaussian error propagation (root mean square).

Uncertainties in the proton energy scale were estimated based on the incident energy spread and the energy deviation deduced from the monitor reactions. This results in a steady increase in uncertainty of about $7 \%$ per degraded $\mathrm{MeV}$ starting with $\pm 0.2 \mathrm{MeV}$ at $45 \mathrm{MeV}$ up to a maximum of $\pm 2 \mathrm{MeV}$ in the last monitor foil at $17 \mathrm{MeV}$ for the irradiation at JULIC. For irradiations at BC1710 the uncertainty ranges from \pm 0.1 at $17.1 \mathrm{MeV}$ up to $\pm 1 \mathrm{MeV}$ in the last monitor foil at $7.7 \mathrm{MeV}$. Therewith the uncertainty in proton energy scale ranges within 0.5 to $13 \%$ from highest to lowest energy.

\subsection{Separation of no-carrier-added products}

A radiochemical separation method for the radionuclide ${ }^{52} \mathrm{Mn}$ produced from bulk chromium and the co-produced Cr-activities was developed. Radiovanadium was not coproduced in the main production energy range of ${ }^{52 \mathrm{~g}, \mathrm{~m}} \mathrm{Mn}$ (5.6 to $20 \mathrm{MeV}$ ), therefore separation from ${ }^{48} \mathrm{~V}$ was not needed. A scheme of the separation procedure is depicted in Fig. 1.

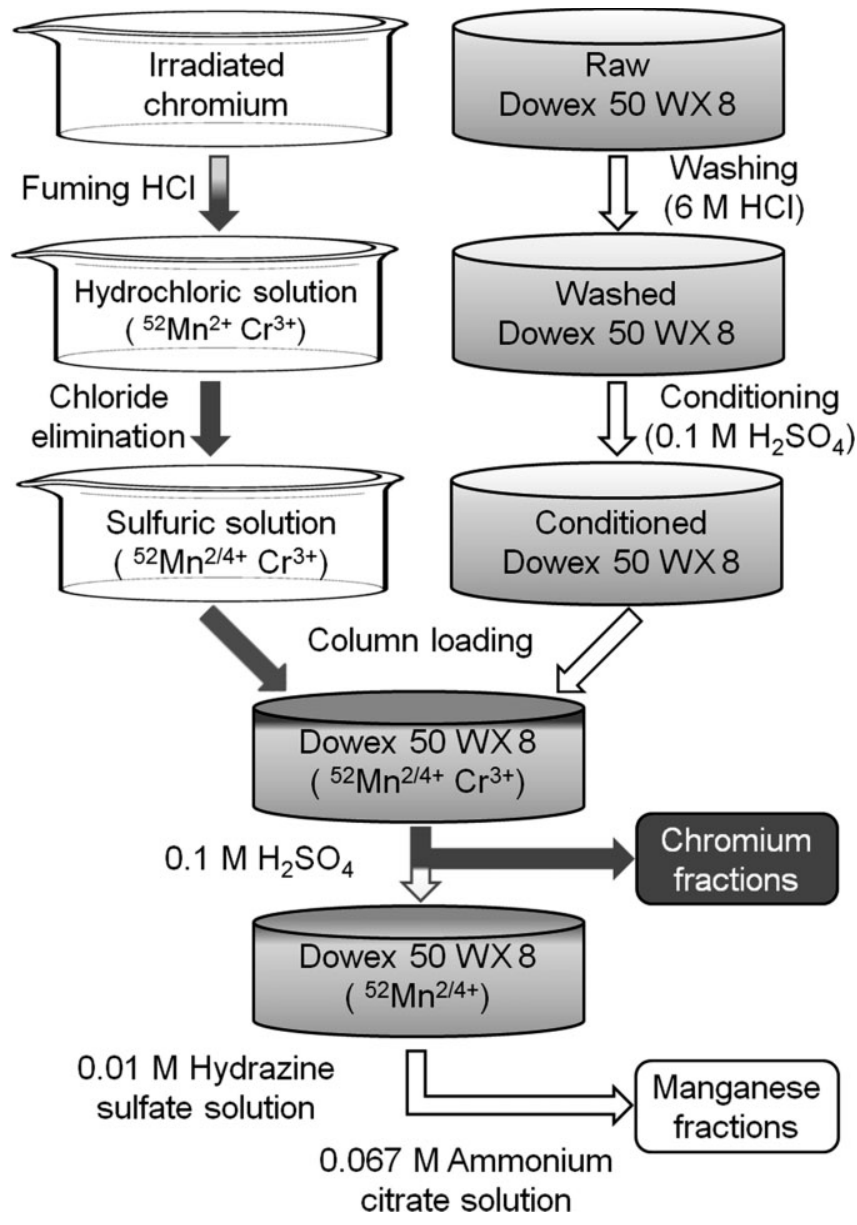

Fig. 1. Scheme of the new separation procedure.
The intended later application of labelled MRI-active agents in animals and humans rules out the use of any highly toxic eluent. Thus, additional time consuming chemical reprocessing would be necessary for most of the presently known separation methods $[8,10,11]$. In order to avoid those, ion exchange chromatography was utilized with an ammonium citrate solution as a non-hazardous, further on applicable eluent. A hint for the development came from a published macroscopic separation of manganese and chromium using the ion exchange resin DOWEX $50 \mathrm{~W} \times 8$ [21] and a publication about the behaviour of manganese on the latter resin [22].

\subsubsection{Irradiated target processing}

For initial separation studies irradiated targets of electrolytically deposited chromium were dissolved in $1 \mathrm{~mL}$ hot, fuming hydrochloric acid at $\approx 70{ }^{\circ} \mathrm{C}$ within 10 min, leaving the gold backing unaffected. No activation products originating from the gold foil were detected in solutions subjected to $\gamma$-ray spectrometric controls. After 5 min cooling time the gold foil was removed with a PE-forceps. For up-scaling the radiochemical separation, a stock solution was prepared after grinding the irradiated chromium discs $(\approx 470 \mathrm{mg})$ in a ball mill (Pulverisette 23, Fritsch). The resulting powder was dissolved in 10-20 mL hot, fuming hydrochloric acid at $\approx 70{ }^{\circ} \mathrm{C}$.

The hydrochloric solutions (1-3 mL) obtained were evaporated to dryness, redissolved in $5 \mathrm{~mL}$ pure water and again heated and evaporated to dryness. The residue was then dissolved in $1 \mathrm{~mL} 1.5 \mathrm{M}$ sulfuric acid and concentrated to approx. $0.2 \mathrm{~mL}$ to eliminate remaining chloride ions. Control of complete chloride elimination by repetition of volume decrease was done by determining the chloride anions using an ion-chromatographic separation system (Methrom, Kompakt-IC 882) connected to a conductance detector on a Metrosep A Supp 5 - 150/4.0 column. A solution of $3.2 \mathrm{mmol} / \mathrm{L} \mathrm{Na}_{2} \mathrm{CO}_{3}$ and $1.0 \mathrm{mmol} / \mathrm{L} \mathrm{NaHCO}_{3}$ in water was applied as chromatographic medium. The purge of chloride ions was mandatory for a successful separation of $\mathrm{Cr}$ and $\mathrm{Mn}$. If chloride ions remained in the solution (detection limit: $0.34 \mu \mathrm{g} / \mathrm{L}$; source: Methrom AG; IC Application Work CH6-1004-072009 2009), a quantitative elution of chromium from the column was not possible. An evaporation of the solutions to dryness is also not possible, due to the formation of $\mathrm{SO}_{3}$ in excess which would cause oxidation of chromium(III) leading to precipitation of undissolvable chromates.

\subsubsection{Chromatographic separation}

For the chromatographic separation of radiomanganese from "bulk" chromium a previously prepared DOWEX $50 \mathrm{~W} \times 8$ ( $\mathrm{H}^{+}$-form, 50-100 mesh size) cation-exchange resin column (10 mm diameter, $50 \mathrm{~mm}$ length) was used. To cleanse the resin from production impurities (mainly iron ions) it was washed prior to use with half-concentrated hydrochloric acid and stored in an air-tight container. The pre-washed resin was then soaked in pure water for at least $24 \mathrm{~h}$ prior to the radiochemical separation and was conditioned with tenfold column volume of $0.1 \mathrm{M}$ sulfuric acid solution. No measureable chloride ions remained in the eluent which was 
confirmed by testing with a $\mathrm{AgNO}_{3}$ solution. The chloride free target solution was diluted with pure water to obtain a $c a$. $0.1 \mathrm{M}$ sulfuric acid solution and then loaded on the prepared DOWEX-column.

In a first step the chromium ions were quantitatively eluted with $40 \mathrm{~mL}$ of $0.1 \mathrm{M}$ sulfuric acid solution. Then the column was washed with $10 \mathrm{~mL}$ pure water, loaded with a solution of $10 \mathrm{~mL}$ of $0.01 \mathrm{M}$ hydrazine sulphate, in order to reduce eventually oxidized radiomanganese back to $\mathrm{Mn}^{2+}$, and finally washed with $10 \mathrm{~mL}$ pure water again. Afterwards the radiomanganese was quantitatively eluted with a solution of $0.067 \mathrm{M}$ ammonium citrate $(\mathrm{pH}=7.3-7.4)$. The collected fraction volumes were varied according to the used eluent for a precise elution curve: $5 \mathrm{~mL}$ fractions for sulfuric acid solution (chromium) and $2.5 \mathrm{~mL}$ for ammonium citrate solution (manganese). The eluted fractions were cautiously reduced to dryness at $90-100{ }^{\circ} \mathrm{C}$. The radioisotopes in the remaining residue were determined for radiochemical purity by $\gamma$-ray spectrometry.

\section{Results and discussion}

\subsection{Experimentally measured cross sections}

\subsubsection{Present literature and new experimental results}

The focus in this study was on ${ }^{52 \mathrm{~g}} \mathrm{Mn}$, due to its more convenient half-life for practical developments like separation procedures and optimization of old or evaluation of new labelling techniques. There are several publications regarding its production. Some of them are outdated (1962/1975) $[23,24]$, because more exact decay or $\gamma$-ray intensity data were published later. These are directly linked to the calculation of cross sections and thick target yields by means of the activation formula. Additionally the maxima of the

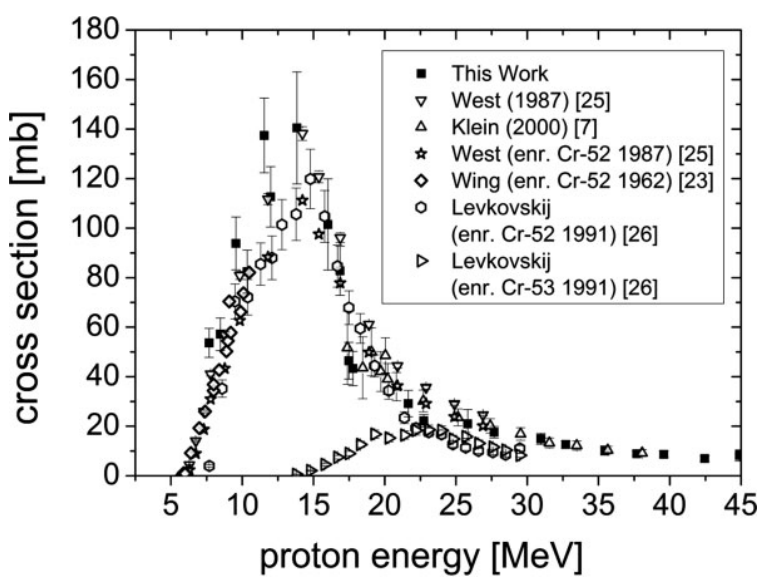

Fig. 2. Experimental cumulative ${ }^{\text {nat }} \mathrm{Cr}(p, x)^{52 \mathrm{~g}} \mathrm{Mn}$ cross sections and literature data. The data for the formation of ${ }^{52 \mathrm{~g}} \mathrm{Mn}$ via the ${ }^{53} \mathrm{Cr}(p, 2 n)$ reaction [26] are also shown.

excitation functions are not well described in those publications $[23,25]$. Concerning the production of ${ }^{51} \mathrm{Mn}$ comprehensive data have been reported by Klein et al. [7],, including additional cross section data for ${ }^{52 \mathrm{~g}, \mathrm{~m}} \mathrm{Mn}$ and the important radioactive by-products ${ }^{48} \mathrm{~V},{ }^{48} \mathrm{Cr}$ and ${ }^{51} \mathrm{Cr}$. However, due to some contradictions in the published data, e.g. for the formation of ${ }^{51} \mathrm{Cr}[7,24]$ verification and extension of all the measured data appeared mandatory.

The measured cross section data for the formation of the radionuclides ${ }^{52 \mathrm{~g}, \mathrm{~m}} \mathrm{Mn},{ }^{48} \mathrm{~V}$ and ${ }^{48,49,51} \mathrm{Cr}$ are given in Table 2. They are also shown in Figs. 2-7 as a function of the proton energy, together with the corresponding literature data [7, 23-29]. For direct comparison the literature cross section data measured on enriched target materials given in the figures were converted to the natural abundance of the respective chromium isotope (see Table 3 ). Due to the use of

Table 2. Measured ${ }^{\text {nat }} \mathrm{Cr}(p, x)^{52 \mathrm{~g}, \mathrm{~m}} \mathrm{Mn} ;{ }^{48} \mathrm{~V} ;{ }^{48,49,51} \mathrm{Cr}$ reaction cross sections.

\begin{tabular}{|c|c|c|c|c|c|c|}
\hline \multirow[t]{2}{*}{ Energy $[\mathrm{MeV}]$} & \multicolumn{6}{|c|}{ Cross section $[\mathrm{mb}]$} \\
\hline & ${ }^{52 \mathrm{~g}} \mathrm{Mn}$ & ${ }^{52 \mathrm{~m}} \mathrm{Mn}$ & ${ }^{48} \mathrm{~V}$ & ${ }^{48} \mathrm{Cr}$ & ${ }^{49} \mathrm{Cr}$ & ${ }^{51} \mathrm{Cr}$ \\
\hline $44.9 \pm 0.2$ & $8.2 \pm 2.3$ & - & - & - & - & $281 \pm 39$ \\
\hline $42.5 \pm 0.4$ & $6.9 \pm 1.1$ & $10.8 \pm 0.8$ & $30.5 \pm 4.5$ & $1.54 \pm 0.12$ & $9.2 \pm 0.6$ & $251 \pm 40$ \\
\hline $39.6 \pm 0.5$ & $8.7 \pm 1.3$ & $8.53 \pm 0.63$ & $39.0 \pm 6.3$ & $1.95 \pm 0.11$ & $8.5 \pm 0.6$ & $274 \pm 25$ \\
\hline $37.7 \pm 0.7$ & $8.9 \pm 1.1$ & $16.7 \pm 1.2$ & $39.7 \pm 2.9$ & $1.29 \pm 0.10$ & $7.9 \pm 0.5$ & $276 \pm 22$ \\
\hline $35.5 \pm 0.8$ & $10.2 \pm 1.8$ & $13.4 \pm 1.0$ & $47.8 \pm 7.9$ & $1.18 \pm 0.11$ & $8.4 \pm 0.8$ & $318 \pm 33$ \\
\hline $32.7 \pm 1.0$ & $12.7 \pm 1.4$ & $19.1 \pm 1.4$ & $63.0 \pm 4.3$ & $0.69 \pm 0.07$ & $10.6 \pm 0.6$ & $407 \pm 34$ \\
\hline $31.0 \pm 1.1$ & $14.9 \pm 2.2$ & $12.1 \pm 0.9$ & $66.6 \pm 11.9$ & - & $12.6 \pm 0.8$ & $472 \pm 35$ \\
\hline $27.7 \pm 1.3$ & $17.6 \pm 2.3$ & $21.3 \pm 1.6$ & $49.0 \pm 5.7$ & - & $13.5 \pm 0.9$ & $551 \pm 45$ \\
\hline $25.8 \pm 1.4$ & $21.0 \pm 5.8$ & $28.8 \pm 2.1$ & $43.1 \pm 3.6$ & - & $13.7 \pm 0.8$ & $569 \pm 53$ \\
\hline $22.7 \pm 1.6$ & $22.2 \pm 2.4$ & $33.7 \pm 2.5$ & $16.6 \pm 1.1$ & - & $10.6 \pm 0.7$ & $535 \pm 72$ \\
\hline $21.7 \pm 1.7$ & $29.1 \pm 5.3$ & $36.7 \pm 2.7$ & $6.7 \pm 1.0$ & - & $11.4 \pm 0.9$ & $505 \pm 57$ \\
\hline $17.8 \pm 1.9$ & $43.3 \pm 7.0$ & $62.5 \pm 4.6$ & - & - & $7.3 \pm 0.5$ & $425 \pm 44$ \\
\hline $17.5 \pm 2.0$ & $46.4 \pm 7.4$ & $61.8 \pm 4.5$ & - & - & $5.8 \pm 0.4$ & $357 \pm 29$ \\
\hline $16.9 \pm 0.1$ & $82.8 \pm 10.0$ & $149 \pm 11$ & - & - & $3.5 \pm 0.4$ & $316 \pm 48$ \\
\hline $16.0 \pm 0.2$ & $102 \pm 18$ & - & - & - & - & - \\
\hline $15.1 \pm 0.3$ & - & $250 \pm 19$ & - & - & - & - \\
\hline $13.8 \pm 0.4$ & $141 \pm 23$ & $380 \pm 27$ & - & - & - & - \\
\hline $12.0 \pm 0.6$ & $113 \pm 12$ & $361 \pm 26$ & - & - & - & - \\
\hline $11.5 \pm 0.6$ & $137 \pm 15$ & - & - & - & - & \\
\hline $10.3 \pm 0.8$ & $82.5 \pm 8.7$ & $349 \pm 25$ & - & - & - & \\
\hline $9.6 \pm 0.8$ & $93.8 \pm 10.7$ & $381 \pm 28$ & - & - & - & \\
\hline $8.5 \pm 0.9$ & $57.2 \pm 6.6$ & $288 \pm 21$ & - & - & - & \\
\hline $7.7 \pm 1.0$ & $53.6 \pm 5.9$ & $267 \pm 20$ & - & - & - & \\
\hline
\end{tabular}




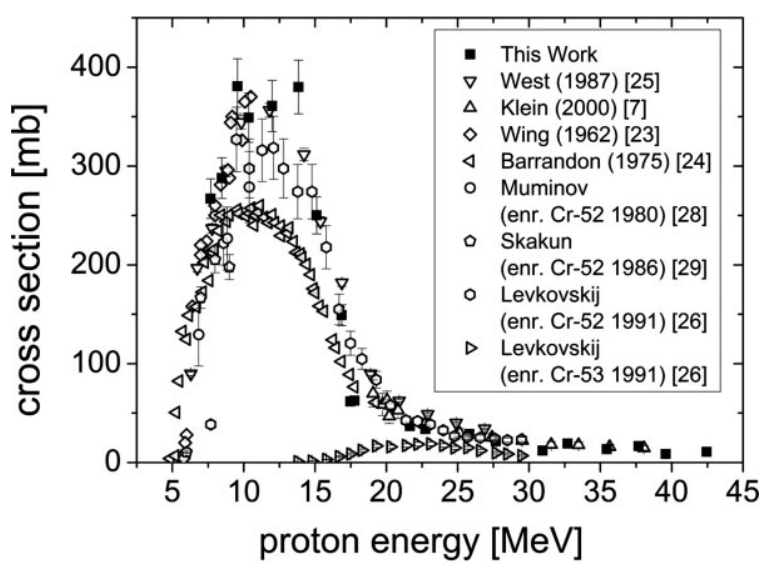

Fig. 3. Experimental ${ }^{\text {nat }} \mathrm{Cr}(p, x)^{52 \mathrm{~m}} \mathrm{Mn}$ cross sections and literature data The data for the formation of ${ }^{52 \mathrm{~m}} \mathrm{Mn}$ via the ${ }^{52} \mathrm{Cr}(p, 2 n)-[26,28,29]$ and ${ }^{53} \mathrm{Cr}(p, 3 n)$-reaction [26] are also shown.

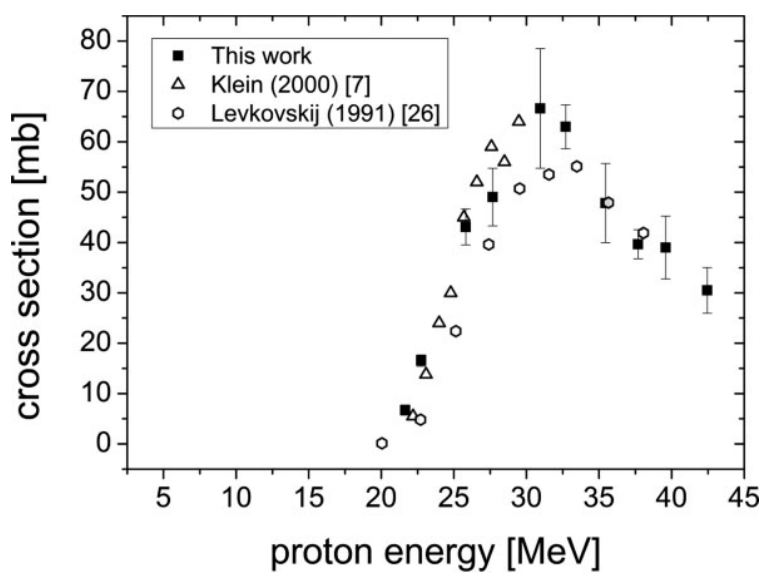

Fig. 4. Experimental ${ }^{\text {nat }} \mathrm{Cr}(p, x)^{48} \mathrm{~V}$ cross sections and literature data.

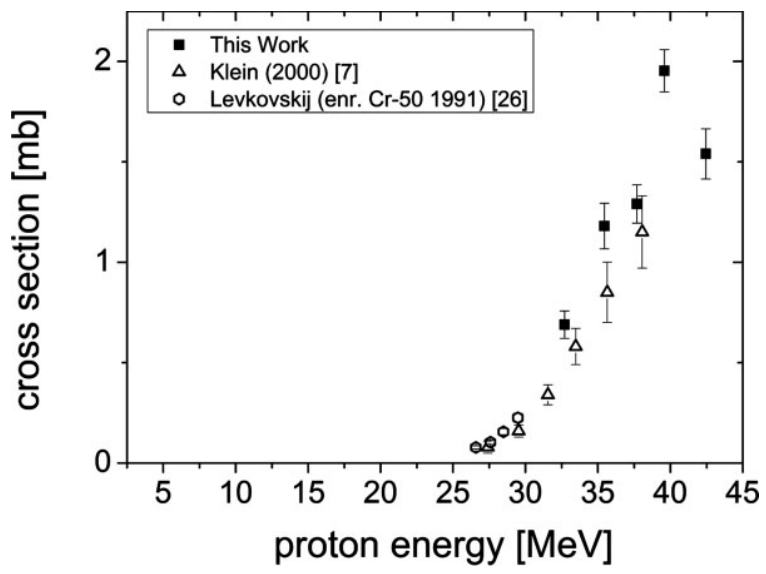

Fig. 5. Experimental ${ }^{\text {nat }} \mathrm{Cr}(p, x)^{48} \mathrm{Cr}$ cross sections and literature data. The data for the formation of ${ }^{48} \mathrm{Cr}$ via the ${ }^{5} \mathrm{Cr}(p, p 2 n)$-reaction [26] are also shown.

natural chromium as target material there are many different reaction channels possible for the formation of the measured radionuclides.

\subsection{2 ${ }^{52 \mathrm{~g}, \mathrm{~m}} \mathrm{Mn}$ cross sections}

The excitation functions of ${ }^{52 g, \mathrm{~m}} \mathrm{Mn}$ (Figs. 2 and 3) represent three production channels (for thresholds see Table 3

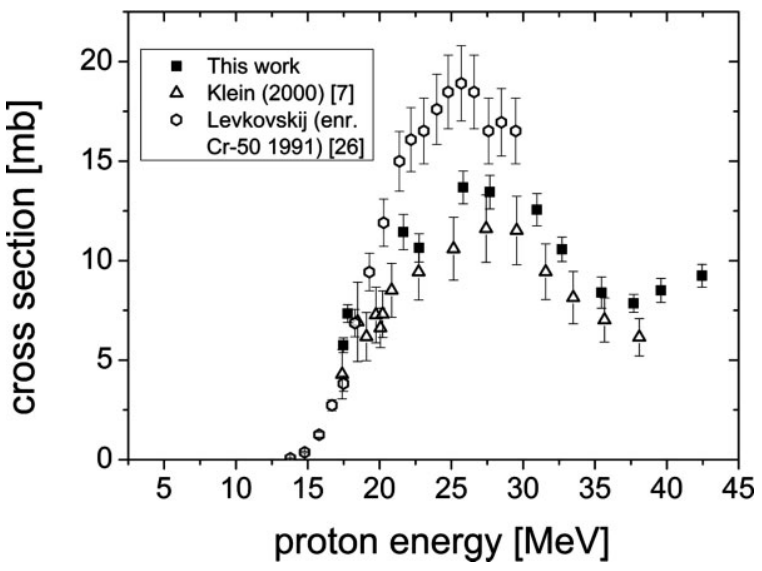

Fig. 6. Experimental ${ }^{\text {nat }} \mathrm{Cr}(p, x)^{49} \mathrm{Cr}$ cross sections and literature data. The data for the formation of ${ }^{49} \mathrm{Cr}$ via the ${ }^{5} \mathrm{Cr}(p, p n)$-reaction [26] are also shown.

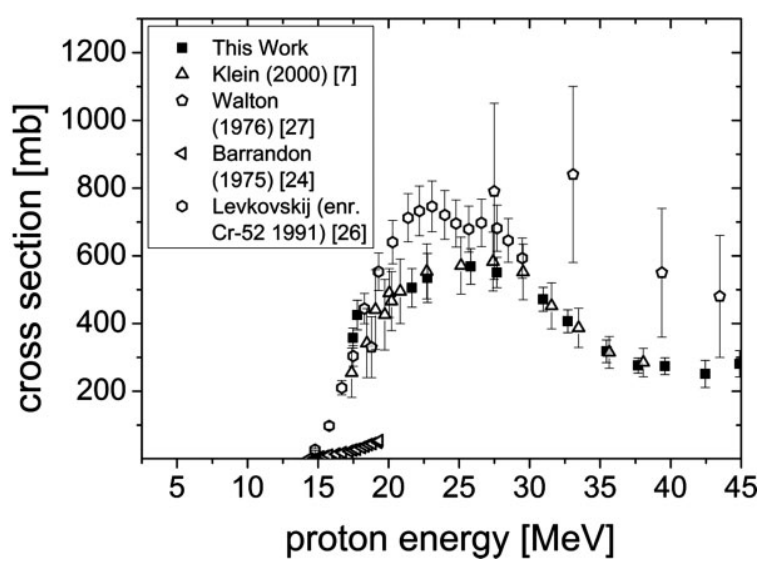

Fig. 7. Experimental nat $\mathrm{Cr}(p, x)^{51} \mathrm{Cr}$ cross sections and literature data. The data for the formation of ${ }^{51} \mathrm{Cr}$ via the ${ }^{52} \mathrm{Cr}(p, p n)$-reaction [26] are also shown.

Table 3. Energy thresholds of nuclear reactions contributing to production of radioisotopes and natural abundance of corresponding chromium isotopes.

\begin{tabular}{|c|c|c|}
\hline $\begin{array}{l}\text { Reaction } \\
\text { channel }\end{array}$ & $\begin{array}{c}\text { Energy threshold } \\
{[\mathrm{MeV}][30]}\end{array}$ & $\begin{array}{l}\text { Natural abundance } \\
\text { in }{ }^{\text {nat }} \mathrm{Cr}[\%][37]\end{array}$ \\
\hline${ }^{52} \mathrm{Cr}(p, n)^{52 \mathrm{~g}} \mathrm{Mn}$ & 5.5 & $83.79 \pm 0.02$ \\
\hline${ }^{52} \mathrm{Cr}(p, n)^{52 \mathrm{~m}} \mathrm{Mn}$ & 5.9 & $83.79 \pm 0.02$ \\
\hline${ }^{53} \mathrm{Cr}(p, 2 n)^{52 \mathrm{~g}} \mathrm{Mn}$ & 13.4 & $9.50 \pm 0.02$ \\
\hline${ }^{53} \mathrm{Cr}(p, 2 n)^{52 \mathrm{~m}} \mathrm{Mn}$ & 13.8 & $9.50 \pm 0.02$ \\
\hline${ }^{54} \mathrm{Cr}(p, 3 n)^{52 \mathrm{~g}} \mathrm{Mn}$ & 23.2 & $2.37 \pm 0.01$ \\
\hline${ }^{54} \mathrm{Cr}(p, 3 n)^{52 \mathrm{~m}} \mathrm{Mn}$ & 23.5 & $2.37 \pm 0.01$ \\
\hline${ }^{50} \mathrm{Cr}(p, p 2 n)^{48} \mathrm{Cr}$ & 23.6 & $4.56 \pm 0.01$ \\
\hline${ }^{50} \mathrm{Cr}(p, p n)^{49} \mathrm{Cr}$ & 13.0 & $4.56 \pm 0.01$ \\
\hline${ }^{52} \mathrm{Cr}(p, p 3 n){ }^{49} \mathrm{Cr}$ & 34.3 & $83.79 \pm 0.02$ \\
\hline${ }^{52} \mathrm{Cr}(p, p n)^{51} \mathrm{Cr}$ & 12.0 & $83.79 \pm 0.02$ \\
\hline${ }^{53} \mathrm{Cr}(p, p 2 n)^{51} \mathrm{Cr}$ & 20.0 & $9.50 \pm 0.02$ \\
\hline${ }^{54} \mathrm{Cr}(p, p 3 n)^{51} \mathrm{Cr}$ & 29.7 & $2.37 \pm 0.01$ \\
\hline${ }^{50} \mathrm{Cr}(p . \mathrm{He}-3)^{48} \mathrm{~V}$ & 21.6 & $4.56 \pm 0.01$ \\
\hline${ }^{52} \mathrm{Cr}(p, \alpha n)^{48} \mathrm{~V}$ & 14.1 & $83.79 \pm 0.02$ \\
\hline${ }^{53} \mathrm{Cr}(p, \alpha 2 n)^{48} \mathrm{~V}$ & 22.1 & $9.501 \pm 0.02$ \\
\hline
\end{tabular}

[30]). The main production route using natural target material is the ${ }^{52} \mathrm{Cr}(p, n)^{52 \mathrm{~g}, \mathrm{~m}} \mathrm{Mn}$ reaction. For a more accurate measurement of the contributing reaction channels, of course experiments with highly enriched targets have to be 


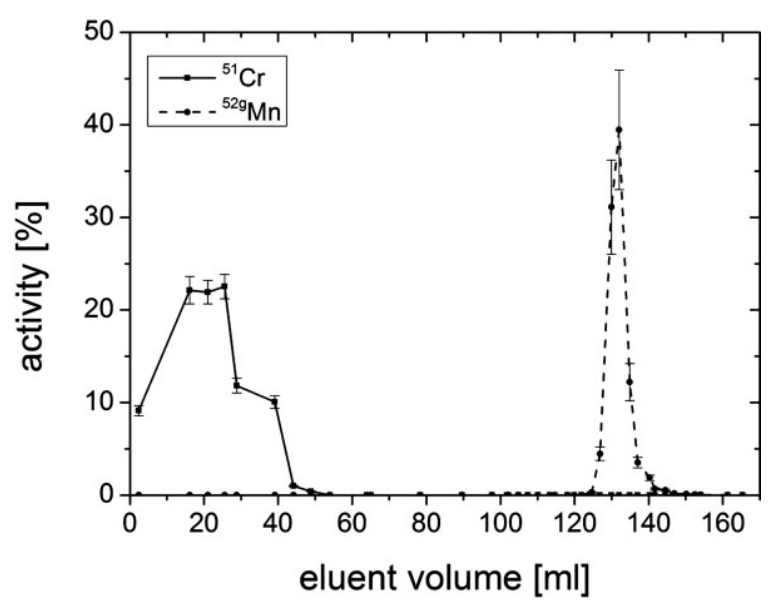

Fig. 8. Elution profile of the newly developed cation-exchange separation. The profile for ${ }^{51} \mathrm{Cr}$ is rather broad due to presence of bulk of Cr.

conducted. The focus of this study was the general production of non-carrier-added ${ }^{52 \mathrm{~g}} \mathrm{Mn}$, therefore this assessment was not necessary here. But it is interesting to notice that the peak maximum of the excitation functions of ${ }^{52 \mathrm{~g}} \mathrm{Mn}$ and ${ }^{52 \mathrm{~m}} \mathrm{Mn}$ are relatively broad which is due to the contribution of different reaction channels. A more detailed discussion of model calculations about the complex dependence of isomeric cross-section ratio of ${ }^{52 \mathrm{~m}, \mathrm{~g}} \mathrm{Mn}$ on different reaction channels can be found in an earlier publication [31].

A general comparison of the experimentally measured cross sections for ${ }^{52 \mathrm{~g}, \mathrm{~m}} \mathrm{Mn}$ with already published data shows a good agreement. The excitation function maxima for both nuclides are between 10 and $15 \mathrm{MeV}$. The heights of the maxima are determined as approximately $140 \mathrm{mb}$ for ${ }^{52 \mathrm{~g}} \mathrm{Mn}$ and $380 \mathrm{mb}$ for ${ }^{52 \mathrm{~m}} \mathrm{Mn}$. Only two data points at $9.6 \mathrm{MeV}$ and $13.8 \mathrm{MeV}$, respectively, appear to be too high. An exception from the agreement are the data for ${ }^{52 \mathrm{~m}} \mathrm{Mn}$ from Barrandon et al. [24] whose divergence is discussed in more detail later on. Smaller deviations can be attributed to the uncertainties which generally influence cross section measurements. Especially the particle flux determination and the detector efficiency contribute to uncertainties which lead to a spreading of data. The fluctuation between the uncertainties of the individual cross sections is caused by varying detection geometries, different detectors used, and the variation of irradiation specifications (irradiation time, proton flux, and measurement time) in each of the irradiations. This leads to individual uncertainties of up to $28 \%$.

\subsection{3 ${ }^{48,49,51} \mathrm{Cr}$ and ${ }^{48} \mathrm{~V}$ cross sections}

The cross sections of the ${ }^{\text {nat }} \mathrm{Cr}(p, p x n){ }^{48} \mathrm{Cr}$ reaction were previously determined only once [7] and are reconfirmed by the present measurements (see Fig. 5). The cross sections rise with a steady slope up to $2 \mathrm{mb}$ at approx. $40 \mathrm{MeV}$. Furthermore the assessed data for the ${ }^{\text {nat }} \mathrm{Cr}(p, p x n){ }^{49,51} \mathrm{Cr}$ reactions are also in agreement with published cross sections [7]. The observed excitation function maxima for the $\left.{ }^{\text {nat }} \mathrm{Cr}(p, p \times n)\right)^{49,51} \mathrm{Cr}$ reactions are located between 20 and $30 \mathrm{MeV}$ with peak values of about $14 \mathrm{mb}$ and $570 \mathrm{mb}$, respectively. The uncorrected cross section data of Levskovskij [26] for ${ }^{52} \mathrm{Cr}(p, x)^{48,49} \mathrm{Cr}$ reactions measured with enriched target materials and converted to natural chromium abundance are on average higher than the results found here. Also data published by Barrandon et al. [24] and Walton et al. [27] show a different trend for the excitation function of the ${ }^{\text {nat }} \mathrm{Cr}(p, p x n)^{51} \mathrm{Cr}$ reaction. Especially the values of Barrandon et al. [24] in the proton energy region from 17.5 to $20 \mathrm{MeV}$ differ largely from the newly measured cross sections. The same trend is again found for the ${ }^{\text {nat }} \mathrm{Cr}(p, x n){ }^{52 \mathrm{~m}} \mathrm{Mn}$ reaction described in the previous section. The difference in the peak area is up to $35 \%$ between the cross sections from Barrandon et al. [24] and our measurements. This strong deviation starts upwards $10 \mathrm{MeV}$ proton energy. This indicates a systematic error in the latter measurements. Therefore and due to the good agreement of all other measured reaction cross sections it appears reasonable that the newly measured data and those published by Klein et al. [7] are more accurate.

With respect to ${ }^{48} \mathrm{~V}$ there is also a good agreement of the excitation function maximum around $30 \mathrm{MeV}$ and its height of about $67 \mathrm{mb}$ with the data given by Klein et al. [7]. Even without the correction of Takács et al. [32] the cross section data by Levskovskij [26] are lower than the cross sections presented here. This is interesting due to the fact that the cross sections for the production of ${ }^{48,49} \mathrm{Cr}$ of Levskovskij [26] are higher than those measured here.

In consideration of threshold energies (see Table 3) the most likely production channel for the formation of ${ }^{48} \mathrm{~V}$ is the ${ }^{50} \mathrm{Cr}(p, x)^{48} \mathrm{~V}$ reaction. But as mentioned before future experiments with enriched target materials are mandatory for a precise interpretation of the production channels.

\subsection{Integral yields}

All experimentally measured production rates, derived saturated thick target yields and theoretically assessed integral yields covering the maximum of the excitation function of the ${ }^{\text {nat }} \mathrm{Cr}(p, x)^{52 \mathrm{~g}, \mathrm{~m}} \mathrm{Mn}$ reaction are shown in Table 4. Some yields found in the literature are also listed in Table 4 $[28,33,34]$. These were arithmetically converted into the saturation thick target yields in $\mathrm{Bq} / \mu \mathrm{A}$ for comparison.

The here measured cumulative saturation yields in the proton energy range of 16.9 to $8.2 \mathrm{MeV}$ amount to $2.55 \pm$ 0.31 and $6.96 \pm 0.57 \mathrm{GBq} / \mu \mathrm{A}$ for the ground state and the metastable state of ${ }^{52} \mathrm{Mn}$, respectively. With the new experimental results the activity contribution of the metastable state to the ground state is approximately $4.7 \%$. The difference between experimental saturation yields and calculated saturation integral yields of ${ }^{52 \mathrm{~g}} \mathrm{Mn}$ and ${ }^{52 \mathrm{~m}} \mathrm{Mn}$ are about 0.19 $(8 \%)$ and $1.07 \mathrm{GBq} / \mu \mathrm{A}(15 \%)$, respectively, representing an acceptable variance within one $\sigma$ for ${ }^{52 \mathrm{~g}} \mathrm{Mn}$ and two $\sigma$ for ${ }^{52 \mathrm{~m}} \mathrm{Mn}$.

In the literature only one determination of the thick target yield for ${ }^{52 \mathrm{~g}} \mathrm{Mn}$ (16 MeV to beam stop) exists for the optimal proton energy production range $(0.98 \mathrm{GBq} / \mu \mathrm{A})$ [33]. This is only $38.5 \%$ of our experimental results and $36.6 \%$ of our theoretical calculations. Other reported thick target yields are either covering a too narrow (12 MeV to beam stop [28]) or a too large energy region (22 MeV to threshold [34]), where more radioactive impurities (e.g. ${ }^{48,49,51} \mathrm{Cr}$ ) are produced. Therefore a comparison of the measured data with those literature values seems not reasonable. 
Table 4. Experimental production rates, calculated saturation thick target yields and respective integral yields of main radioisotopes produced.

\begin{tabular}{|c|c|c|c|c|c|c|c|}
\hline \multirow[t]{2}{*}{ Isotope } & \multirow{2}{*}{$\begin{array}{l}\text { Production rate } \\
{\left[\mathrm{Bq} \mu \mathrm{A}^{-1} \mathrm{~h}^{-1}\right]^{a}}\end{array}$} & \multirow{2}{*}{$\begin{array}{l}\text { Integral yield } \\
{\left[\mathrm{Bq} \mu \mathrm{A}^{-1} \mathrm{~h}^{-1}\right]^{b}}\end{array}$} & \multirow{2}{*}{ 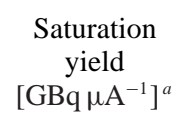 } & \multirow{2}{*}{ 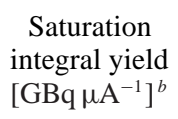 } & \multicolumn{3}{|c|}{ Saturation yield $\left[\mathrm{GBq} \mu \mathrm{A}^{-1}\right]$} \\
\hline & & & & & $\begin{array}{l}\text { Munimov [28] } \\
\quad(12 \mathrm{MeV})\end{array}$ & $\begin{array}{l}\text { Abe [33] } \\
(16 \mathrm{MeV})\end{array}$ & $\begin{array}{l}\text { Dmitriev [34] } \\
\quad(22 \mathrm{MeV})\end{array}$ \\
\hline${ }^{52 g} \mathrm{Mn}$ & $(1.31 \pm 0.16) \times 10^{7}$ & $(1.37 \pm 0.16) \times 10^{7}$ & $2.55 \pm 0.31$ & $2.74 \pm 0.30$ & - & 0.98 & $4.07 \pm 0.47$ \\
\hline${ }^{52 \mathrm{~m}} \mathrm{Mn}$ & $(5.99 \pm 0.49) \times 10^{9}$ & $(6.91 \pm 0.76) \times 10^{9}$ & $6.96 \pm 0.57$ & $8.03 \pm 0.88$ & 7.25 & - & - \\
\hline${ }^{51} \mathrm{Cr}$ & $(1.60 \pm 0.16) \times 10^{6}$ & $(1.73 \pm 0.19) \times 10^{6}$ & $1.53 \pm 0.15$ & $1.66 \pm 0.18$ & - & - & $5.66 \pm 0.67$ \\
\hline
\end{tabular}

a: for $E_{\mathrm{P}}=16.9 \rightarrow 8.2 \mathrm{MeV}$ (experimental value);

b: for $E_{\mathrm{P}}=16.9 \rightarrow 8.2 \mathrm{MeV}$ (value calculated from excitation function).

\subsection{Separation of no-carrier-added ${ }^{52} \mathrm{Mn}$ from the "bulk" of chromium}

According to the method described above ${ }^{52 \mathrm{~g}} \mathrm{Mn}$ was quantitatively separated from the "bulk" target material chromium without the addition of an isotopic carrier. The separation technique was repeated in several test runs with identical parameters leading to a main ${ }^{52 \mathrm{~g}} \mathrm{Mn}$ fraction, varying between 10 and $15 \mathrm{~mL}$ eluent. Exemplarily the elution profile of an up-scaled separation $(\approx 225 \mathrm{mg}$ target material) is depicted in Fig. 8. About $99 \%$ of the radiomanganese was found in approximately $12 \mathrm{~mL}$ of the used eluent which had nearly neutral $\mathrm{pH}$ of 7.3-7.4. Only low concentrations of added ammonium citrate $(0.067 \mathrm{M})$ were needed for the separation. Ammonium citrate is non-hazardous in low concentrations and approved as food additive by the European Union (E 380, decree (EU) No. 1129/2011 from 11.11.2011).

Further optimization of the chromatographic separation parameters, i.e. elution speed and eluent concentration was not carried out here, since the research goal of a quantitative separation with a non-hazardous eluent was already met, but it would possibly lead to an even smaller volume of the target fraction.

\subsubsection{Target processing}

The main problem of the radiochemical separation is the time consuming step of chloride-ion elimination. With the macroscopic separation [21] the typical formation of $\mathrm{SO}_{3}$ haze was used as indicator for an adequate decrease of volume. In the present separation the volumes of sulfuric acid were too low to recognize the formation of a characteristic $\mathrm{SO}_{3}$ haze. In order to optimize the time requirement for the chloride elimination a quantitation of the anions was conducted in defined time intervals. Nevertheless, the separation still took 3 to $4 \mathrm{~h}$. With respect to an upscaling of the production using higher amounts of target material, the volume reduction step had to be repeated several times in order to achieve a sufficiently low chloride concentration.

The search for an alternative removal process of chloride was not successful. A change of the dissolving agent is not possible with electro-deposited chromium. Corresponding literature points out that only non-passivated chromium would be dissolvable in diluted, non-oxidizing acids [35]. However, by using the electro-depositing method only passivated elemental chromium is accessible. Even by anodic activation it was not possible to dissolve the electro-deposited chromium in diluted, non-oxidizing acids.

Precipitation of chloride was no option, because of the interference of nitrate with the actual separation and the vo- luminous form of the precipitate resulting in co-precipitation of radiomanganese. An optimized form of anion-exchange chromatography could be the answer for the successful removal of chloride. In preliminary tests a reduction of chloride ions by roughly $95 \%$ was observed with an acetate loaded DOWEX $1 \mathrm{~W} \times 8$ resin. Due to the low affinity of acetate for the exchange resin, however, the conditioning was very time consuming and not practical. The resin had to be rinsed with 2-3 L 8.7 M acetic acid (half-conc.) over several days to achieve a low chloride concentration, but even then chloride could still be found in the eluent. In the literature anions with a higher affinity to the resin are described for removal of chloride [36], but they proved to be not suitable for the purpose. Hydroxide lead to precipitation of chromium hydroxide, while nitrate interfered with the later separation and had to be eliminated prior to a separation which again would cause a longer preparation time.

A possible solution for circumvention of chloride removal could be the change to a different target system, e.g. chromium salts, adapting the separation method elaborated here. Another method could be the substitution of the sulfuric acid solution by an oxalic acid solution for the elution of chromium. In fact preliminary test showed that more chloride is tolerated with oxalic acid solution during a quantitative elution of chromium. However, the change also resulted in lower separation factors of radiomanganese and chromium. Therefore this subject again deserves further optimization.

\section{Conclusion}

The newly measured cross sections of proton-induced reactions on natural chromium, namely ${ }^{\text {nat }} \mathrm{Cr}(p, x){ }^{48} \mathrm{~V},{ }^{48,49,51} \mathrm{Cr}$, and ${ }^{52 \mathrm{~g}, \mathrm{~m}} \mathrm{Mn}$, strengthen the available data base over a large range of proton energy. In the case of ${ }^{51} \mathrm{Cr}$ and ${ }^{52 \mathrm{~m}} \mathrm{Mn}$ they strongly support a previous measurement by Klein et al. [7], but contradict other published data [24]. In addition, the measurements show low amounts of isotopic impurities in the production range of the positron emitter ${ }^{51} \mathrm{Mn}$ above its threshold energy of $16.5 \mathrm{MeV}$.

${ }^{51} \mathrm{Mn}$ is also an attractive candidate for PET, due to its favorable decay properties (46.2 min half-life; 97\% $\beta^{+}$. emission; $2.2 \mathrm{MeV} \beta^{+}$-energy; $>0.3 \% \gamma$-rays). As mentioned in the introduction its production issues were discussed earlier in detail [7-9]. So far only one authentic labeling of an MRI contrast agent has been realized with ${ }^{51} \mathrm{Mn}$ [9] demonstrating the possibility and potential of authentic radioactive labeling. 
Additionally, the developed radiochemical separation procedure is an alternative to already established separations, resulting in pure n.c.a. radiomanganese in a non-toxic and less aggressive medium within $4 \mathrm{~h}$ of separation time. Additional optimization appears warranted, which may even lead to shorter separation times and smaller volumes needed for a complete harvesting of radiomanganese.

Acknowledgment. The authors thank all cyclotron operators and technicians involved in the irradiations. We are also grateful for the support and advice of Prof. Dr. Syed M. Qaim during this work.

\section{References}

1. Kolb, A., Wehrl, H. F., Pichler, B. J.: Eur. Radiol. 22(8), 17761788 (2012).

2. Marckmann, P., Thomsen, H. S.: J. Am. Soc. Nephrol. 17(9), 2359-2362 (2006).

3. Thomsen, H. S.: Radiol. Clin. North Am. 47(5), 827-831 (2009).

4. Chan, K. W., Wong, W.-T.: Coord. Chem. Rev. 17, 2428-2451 (2007).

5. Michalke, B., Halbach, S., Nischwitz, V.: J. Environ. Monit. 9, 650-656 (2007).

6. Klein, A. T. J., Rösch, F., Qaim, S. M.: J. Electrochem. Soc. 146(12), 4526-4534 (1999).

7. Klein, A. T. J., Rösch, F., Qaim, S. M.: Radiochim. Acta 88, 253-264 (2000).

8. Klein, A. T. J., Rösch, F., Coenen, H. H., Qaim, S. M.: Radiochim. Acta 90, 167-177 (2002).

9. Klein, A. T. J., Rösch, F., Coenen, H. H., Qaim, S. M.: Appl. Radiat. Isotopes 62, 711-720 (2005).

10. Lahiri, S., Nayak, D., Korschinek, G.: Anal. Chem. 78, 7517-7521 (2006).

11. Mttdrová, B., Kukula, F.: Isot. Environ. Health Stud. 3(2), 51-53 (1967).

12. Fessler, A., Alfassi, Z. B., Qaim, S. M.: Radiochim. Acta 65, 207-213 (1994).

13. Mushtaq, A., Qaim, S. M.: Radiochim. Acta 50, 27-39 (1990).

14. Rösch, F., Qaim, S. M., Stöcklin, G.: Radiochim. Acta 61, 1-8 (1993).

15. Shehata, M.: Radiochemical Studies Relevant to Cyclotron Production of Radionuclides ${ }^{71,72} \mathrm{As},{ }^{68} \mathrm{Ge} /{ }^{68} \mathrm{Ga}$ and ${ }^{76,77,80 \mathrm{~m}} \mathrm{Br}$. $\mathrm{PhD}$ Thesis, Universität zu Köln (2011).

16. Tárkányi, F. T., Takács, S., Gul, K., Hermanne, A. Mustafa, M. G., Nortier, M., Obložinský, P., Qaim, S. M., Scholten, B., Shubin, Yu. N., Zhuang, Y.: Monitor reactions. In: Charged Particle
Cross Section Database for Medical Radioisotope Production. IAEA-TECDOC-1211, 49-152, International Atomic Energy Agency, Vienna, (2001).

17. Blessing, G., Bräutigam, W., Böge, H. G., Gad, N., Scholten, B., Qaim, S. M.: Appl. Radiat. Isot. 46(9), 955-960 (1995).

18. Livingston, M. S., Bethe, H. A.: Rev. Mod. Phys. 9, 245-390 (1937).

19. Williamson, C. F., Boujot, J.-B., Picard, J.: Tables of Range and Stopping Power of Chemical Elements for charged Particels of Energy 0.05 to $500 \mathrm{MeV}$, Department de Physique Nucleaire Rapport CEA-R 3042 (1966).

20. Evaluated Nuclear Structure Data File (ENSDF). Available at: http://www.nndc.bnl.gov/ensdf/, IAEA-NDS (2011).

21. Schmitt, B. F., Segebade, C.: Z. Anal. Chem. 270, 193-198 (1974).

22. Akki, S. B., Khopkar, S. M.: Chromatographia 3, 363-365 (1970).

23. Wing, J., Huizenga, J. R.: Phys. Rev. 128, 1, 280-290 (1962).

24. Barrandon, J. N., Debrun, J. L., Kohn, A., Spear, R. H.: Nucl. Instrum. Methods 127, 269-278 (1975).

25. West Jr., H. I., Lanier, R. G., Mustafa, M. G.: Phys. Rev. C 35(6), 2067-2076 (1987).

26. Levkovskij, V. N.: Activation Cross Sections for the Nuclides of Medium Mass Region $(A=40-100)$ with Medium Energy ( $E=10-50 \mathrm{MeV}$ ) Protons and Alpha Particles (Experiment and Systematics). Inter-Vesi, Moscow, Russia (1991).

27. Walton, J. R., Heymann, D., Yaniv, A., Edgerley, D., Rowe, M. W. J. Geophys. Res. 81(32), 5689-5699 (1976).

28. Muminov, A. V., Mukhamedov, S., Vasidov, A.: Sov. At. Energ. 49, 540 (1980).

29. Skakun, E. A., Batij, V. G., Rakivnenko, Ju. N., Rastrepin, O. A.: Bull. Russ. Acad. Sci.-Phys. 50(10), 169 (1986).

30. Lederer, C. M., Shirley, V. S. (eds.): Table of Isotopes. $7^{\text {th }}$ Edn., John Wiley \& Sons INC., New York (1978).

31. Qaim, S. M., Sudár, S., Fessler, A.: Radiochim. Acta 93, 503-506 (2005).

32. Takács, S., Tárkányi, F., Sonck, M., Hermanne, A.: Nucl. Instrum. Methods B 198, 183-196 (2002).

33. Abe, K., Iizuka, A., Hasegawa, A., Morozumi, S.: J. Nucl. Mater. 123, 1-3, 972 (1984).

34. Dmitriev, P. P.: Vop. At. Nauki i Tekhn., Ser. Yadernye Konstanty 2, 57 (1983).

35. Riedel, E., Janiak, C.: Anorganische Chemie. $8^{\text {th }}$ Edn., de Gruyter (2011), p. 807.

36. Korkisch, J.: Handbook of Ion Exchange Resins: Their Application to Inorganic Analytical Chemistry. CRC Press (1989), vol. 6 , p. 293.

37. Rosman, K. J. R., Taylor, P. D. P.: Pure Appl Chem. 70(1), $217-$ 235 (1998). 